\title{
Doenças oculares em neonatos
}

\author{
Oculardiseases in neonates
}

\author{
Daniela Endriss ${ }^{1}$ \\ Liana Maria V.O. Ventura ${ }^{2}$ \\ José Ricardo Diniz ${ }^{3}$ \\ Ana Carolina Celino ${ }^{1}$ \\ Jana Toscano ${ }^{1}$
}

\section{RESUMO}

Objetivo: Identificar as principais doenças oculares numa população de neonatos assistidos pelo Sistema Único de Saúde em maternidades de referência no Estado de Pernambuco, orientando o seguimento e tratamento necessário. Métodos: Realizou-se estudo de corte transversal numa população de recém-nascidos em três maternidades públicas, no período de abril a outubro de 2000. Procedeu-se ao exame oftalmológico durante visitas semanais, orientando-se o seguimento e tratamento dos casos com alterações oculares ou fatores de risco. Resultados: Examinaram-se 3280 recém-nascidos: 1403 (42,8\%) na Maternidade da Encruzilhada(CISAM), 1232 (37,5\%) na Maternidade do Hospital Barão de Lucena (MHBL) e 645 (19,7\%) na Maternidade do Hospital Agamenon Magalhães (MHAM). 387 eram pré-termo ( $11,8 \%$ dos casos). Encaminharam-se ao serviço especializado 701 neonatos $(21,4 \%$ do total examinado) com alterações oculares ou com fatores de risco. Destes, $46,4 \%$ eram pré-têrmo e $37,9 \%$ receberam oxigenoterapia, observando-se maior número porcentual na $\operatorname{MHBL}(45,4 \%)$. Observou-se conjuntivite em 3,0\% dos olhos, leucocoria em $0,4 \%$ e hemorragia subconjuntival em 2,0\%. À fundoscopia, encontraram-se hemorragias retinianas em $7,8 \%$ dos casos, com acometimento macular em 4,3\%. Conclusões: As doenças oculares mais frequientes foram: hemorragias retinianas e conjuntivites. Os principais fatores de risco observados foram: prematuridade e doenças infecciosas neonatais. Os autores enfatizam que o exame ocular deve ser realizado rotineiramente nos neonatos.

Descritores: Oftalmopatias/patologia; Olho/patologia; Recém-nascido; Fatores de risco

\section{INTRODUÇÃ̃O}

Segundo dados da Organização Mundial de Saúde (OMS) existem no mundo mais de 20 milhões de cegos, sendo que 2/3 destes casos são determinados por causas preveníveis ${ }^{(1)}$. Em 1992, estimativas mundiais apontavam para a alta cifra de 1,5 milhão o número de cegos menores de 16 anos, e destes, em torno de $90 \%$ viviam em países em desenvolvimento ${ }^{(2-3)}$. Os transtornos no desenvolvimento da visão afetam o processo de aprendizagem da criança e sua integridade, tanto física como psíquica, sendo fundamental a prevenção e sua identificação precoce ${ }^{(4)}$.

Os programas de saúde pública em oftalmologia devem priorizar as ações relacionadas a prevenção da cegueira e da incapacidade visual, promoção de saúde ocular, organização de assistência oftalmológica e reabilitação de deficientes visuais ${ }^{(3,5)}$. Para o planejamento e realização dessas ações, faz-se mister o conhecimento objetivo da realidade para a qual se destinam. Constata-se, portanto, a necessidade de se conhecerem as causas de perda visual na população infantil, bem como sua distribuição geográfica, como requisito para o desenvolvimento desses programas ${ }^{(2-3,5)}$. 
Nos Estados Unidos cerca de 8,0\% do total de cegos são crianças $^{(6)}$. Catarata congênita, retinopatia da prematuridade e atrofia do nervo óptico foram identificadas como causas freqüentes de cegueira ${ }^{(6)}$.

A catarata congênita é uma causa importante de cegueira em todos os países, constituindo a principal causa de cegueira na América Latina ${ }^{(1,6-9)}$. No Brasil, figura entre as mais freqüentes em praticamente todos os estudos de deficiência visual em crianças, entretanto o resultado visual pós-cirurgia de catarata, na grande maioria dos casos, é pobre em decorrência do diagnóstico e tratamento tardio ${ }^{(1,8,10)}$.

A retinopatia da prematuridade está entre as primeiras causas de cegueira infantil, tanto em países desenvolvidos quanto nos países em desenvolvimento. A assistência neonatal permite a sobrevivência de muitos recém-nascidos com baixo peso e prematuros, que formam o principal grupo de risco para a ocorrência da doença e suas complicações ${ }^{(3,11)}$.

Nos países em desenvolvimento observa-se elevada incidência de conjuntivite neonatorum (primeiro mês de vida), podendo levar à cegueira. Os agentes usados profilaticamente para prevenir esta condição não são completamente efetivos e não são disponíveis em várias partes do mundo ${ }^{(12-13)}$.

Os autores realizaram este estudo com o objetivo de identificar as principais doenças oculares numa população de neonatos assistidos pelo Sistema Único de Saúde em maternidades de referência do Estado de Pernambuco, orientando o seguimento e tratamentos necessários o mais precoce possível. Os autores analisaram as diferenças existentes entre os três serviços.

\section{MÉTODOS}

Realizou-se um estudo de corte transversal em neonatos internados nas enfermarias, berçários e unidades de tratamento intensivo de três maternidades públicas de referência no Estado de Pernambuco: Maternidade da Encruzilhada (CISAM), Maternidade do Hospital Agamenon Magalhães (HAM) e Maternidade do Hospital Barão de Lucena (HBL). O estudo foi realizado durante o período de abril a outubro de 2000, e foram incluídos todos os recém-nascidos com menos de uma semana de vida, após autorização da comissão de ética médica de cada instituição.

Para a execução do projeto contou-se com uma equipe multidisciplinar composta por oftalmologistas pediátricas da Fundação Altino Ventura (FAV), uma pediatra (voluntária), alunos do curso de especialização, de residência médica e do curso de "fellow" em oftalmologia pediátrica da FAV, enfermeiras, técnicos em informática e um estatístico.

Através de um protocolo previamente elaborado, obtiveram-se dados gerais sobre todos os recém-nascidos examinados como peso, sexo, idade gestacional, tipo de parto e realização do exame pré-natal. O exame oftalmológico constava de: inspeção, teste de Bruckner e mapeamento de retina. Para a fundoscopia instilou-se uma gota de tropicamida a $1 \%$, diluída a $50 \%$ com colírio lubrificante ocular.
Nos casos detectados com alterações oculares ou fatores de risco para doença ocular (prematuridade, baixo peso ao nascer, ou antecedentes maternos de infecção gestacional), preencheu-se um segundo protocolo constando de informações detalhadas: dados do exame pré-natal (número de consultas, realização de exames, intercorrências), história familiar e dados neonatais (como Apgar, uso de oxigênio, peso ao nascimento, tipo de parto e idade gestacional). As informações neonatais foram obtidas do prontuário do recém-nascido.

Os casos com conjuntivite submeteram-se a higiene ocular com soro fisiológico a $0,9 \%$, e quando sugestivos de processos bacterianos, fornecia-se gratuitamente e orientava-se a terapêutica com colírio de tobramicina.

Orientaram-se as genitoras quanto à importância do seguimento dos casos com alterações oculares ou fatores de risco para doença ocular, sendo os mesmos encaminhados e examinados no ambulatório especializado da Fundação Altino Ventura, onde foi instituído o tratamento necessário.

Todos os recém-nascidos com menos de 37 semanas de vida e/ou com peso inferior a 2000 gramas (g), independente do uso de oxigenoterapia, foram encaminhados ao ambulatório de retinopatia da prematuridade.

Para análise estatística dos dados, utilizou-se o Software minitab, versão 11.

\section{RESULTADOS}

Examinaram-se 3280 recém-nascidos, distribuídos nas três maternidades visitadas, ocorrendo o maior número de casos examinados na maternidade do CISAM (1403 casos, 42,8\%). Mil seiscentos e setenta e nove neonatos $(51,2 \%)$ eram do sexo masculino e $1601(48,2 \%)$ do sexo feminino. Mil novecentos e setenta crianças $(60,1 \%)$ nasceram de parto transpelviano e $1200(36,6 \%)$ de parto cesariano. Em 110 casos (3,3\%) não se obtiveram dados quanto ao tipo de parto. Eram a têrmo $87,6 \%$ dos neonatos ( 2874 casos com idade $\geq 37$ semanas), 387 $(11,8 \%)$ eram pré-têrmo e $19(0,6 \%)$ eram pós-têrmo. Observouse maior percentagem de recém-nascidos prematuros no HBL, ocorrendo em 13,3\% do total dos casos examinados nessa instituição.

Do total de casos examinados, $701(21,4 \%)$ apresentaram alguma alteração ocular ou suspeita de doença ocular, sendo então encaminhados para seguimento e possível tratamento na Fundação Altino Ventura. Destes, 368 (52,5\%) eram do sexo feminino e $333(47,5 \%)$ do masculino. O peso ao nascimento variou de $700 \mathrm{~g}$ a $4690 \mathrm{~g}$, média de $2651 \mathrm{~g}$, mediana de 2710 g. Quanto à idade gestacional, 370 recém-nascidos $(52,8 \%)$ eram a têrmo, $325(46,4 \%)$ eram pré-têrmo $(<37$ semanas de vida) e seis $(0,8 \%)$ eram pós-têrmo ( $\geq 42$ semanas). Cento e quarenta recém-nascidos prematuros $(43,1 \%)$ encaminhados eram procedentes da maternidade do HBL. Duzentos e sessenta e seis recém-nascidos $(37,9 \%)$ receberam algum tipo de oxigenoterapia, observando-se maior número porcentual na maternidade do HBL $(45,4 \%)$ (Tabela 1$)$. 


\begin{tabular}{|c|c|c|c|c|c|c|}
\hline \multirow[t]{2}{*}{ Maternidade } & \multicolumn{2}{|c|}{$\begin{array}{l}\text { Freqüência de } \\
\text { prematuridade* }^{*}\end{array}$} & \multicolumn{2}{|c|}{ Oxigenoterapia } & \multicolumn{2}{|c|}{$\begin{array}{c}\text { Freqüência de } \\
\text { neonatos }\end{array}$} \\
\hline & $\mathbf{N}$ & $\%$ & $\mathbf{N}$ & $\%$ & $\mathbf{N}$ & $\%$ \\
\hline CISAM & 135 & 41,5 & 117 & 36,0 & 325 & 46,4 \\
\hline $\mathrm{HBL}$ & 140 & 56,2 & 113 & 45,4 & 249 & 35,5 \\
\hline HAM & 50 & 39,4 & 34 & 26,8 & 127 & 18,1 \\
\hline Total & 325 & 46,4 & 266 & 37,9 & 701 & 100,0 \\
\hline \multicolumn{7}{|c|}{${ }^{*}$ Idade gestacional $<37$} \\
\hline
\end{tabular}

Quatrocentos e noventa e cinco crianças (70,6\%) nasceram de parto transpelviano, tendo sido utilizado fórceps em 12 destes casos (2,4\%). A cirurgia cesariana foi realizada em 206 genitoras (29,4\%). O índice de Apgar no primeiro e quinto minutos de vida foi maior ou igual a oito em $391(55,8 \%)$ e 556 neonatos $(79,3 \%)$ respectivamente. Não foi identificado o índice de Apgar em 89 casos (12,7\%).

Nos antecedentes pré-natais, 502 pacientes $(71,6 \%)$ realizaram três ou mais consultas pré-natais com o médico obstetra. Cento e vinte e quatro genitoras $(17,7 \%)$ possuíam história de diabetes e $170(24,3 \%)$ de hipertensão arterial sistêmica. Cento e quarenta e nove $(21,3 \%)$ referiram algum tipo de doença infecciosa no período gestacional sendo vulvovaginites e infecção do trato urinário as mais freqüentemente encontradas, ocorrendo em 110 casos, 15,7\% do total de parturientes encaminhadas. Sífilis foi identificada no período gestacional em 31 pacientes $(4,4 \%)$, das quais $27(87,0 \%)$ eram do CISAM. Duas parturientes $(0,3 \%)$ referiram história de rubéola, duas $(0,3 \%)$ referiram dengue, duas $(0,3 \%)$ referiram varicela e duas $(0,3 \%)$ eram portadoras do vírus da imunodeficiência adquirida (Tabela 2).

As alterações encontradas no exame oftalmológico dos recém-nascidos incluíam quadro de conjuntivite infecciosa em 43 olhos $(3,0 \%)$, leucocoria com catarata congênita em cinco olhos $(0,4 \%)$ e hemorragia subconjuntival em 28 olhos $(2,0 \%)$. Em dois pacientes $(0,3 \%)$ observou-se blefarofimose. O reflexo vermelho pupilar esteve ausente em 24 casos $(3,4 \%)$. Na fundoscopia, a hemorragia no pólo posterior da retina foi a alteração mais freqüentemente encontrada, ocorrendo em 255 recém-nascidos $(7,8 \%)$, dos quais identificou-se acometimento macular em $11(4,3 \%)$ casos (Tabela 3$)$. Trezentos e setenta $(52,8 \%)$ recém-nascidos apresentaram o exame fundoscópico normal, e em 32 (4,6\%) casos não foi possível examinar o fundo do olho por dificuldades como má dilatação pupilar, edema palpebral, opacidades cristalinianas, choro intenso do neonato, e casos graves com instabilidade clínica, inviabilizando o exame do neonato.

\section{DISCUSSÃO}

Nos países plenamente desenvolvidos, as alterações oculares mais comuns em crianças são detectadas na infância precoce. No Reino Unido, o exame ocular é realizado rotineiramente nos recém-nascidos, visando promover o mais precoce possível, adequada orientação terapêutica, aconselhamento genético e outras condutas de suporte às doenças oculares detectadas $^{(7)}$.

Nos países em desenvolvimento, os escassos recursos destinados à área de saúde devem atender prioridades múltiplas e distintas, nem sempre privilegiando programas preventivos $^{(14)}$. Ressalta a escassez de informações na literatura nacional quanto ao exame oftalmológico do recém-nato, visando a identificação precoce de doenças oculares. Existe carência de publicações que mostrem quais as doenças mais prevalentes que determinam perda visual em nosso meio ${ }^{(15)}$.

O pediatra tem um papel muito importante na prevenção da catarata congênita e conseqüentemente da cegueira na infância, porém, freqüentemente ele não possui ou não conhece as informações básicas sobre o assunto ${ }^{(16)}$. É necessário, portanto, que se possa difundir entre pediatras, neonatologistas e até obstetras, a importância da detecção precoce desta doença, que com freqüência, é descoberta por acaso ${ }^{(1)}$. É indispensável se esclarecer quanto à urgência do tratamento e a necessidade de um exame ocular no recém-nato, principalmente diante de qualquer suspeita de alteração ocular ${ }^{(1,8)}$.

A catarata congênita constitui a causa mais freqüente de cegueira evitável na infância, podendo atingir um porcentual de 10 a $15 \%{ }^{(17)}$. A sua importância está, principalmente, na necessidade de tratamento cirúrgico e correção visual o mais cedo possível, além de estar presente em várias desordens

\begin{tabular}{|c|c|c|c|c|c|c|c|c|}
\hline \multirow{2}{*}{$\begin{array}{l}\text { Doenças } \\
\text { infecciosas }\end{array}$} & \multicolumn{2}{|c|}{ CISAM } & \multicolumn{2}{|c|}{ HBL } & \multicolumn{2}{|r|}{ HAM } & \multicolumn{2}{|c|}{ Freqüência } \\
\hline & $\mathbf{N}$ & $\%$ & $\mathbf{N}$ & $\%$ & $\mathbf{N}$ & $\%$ & $\mathbf{N}$ & $\%$ \\
\hline ITU/VV* & 36 & $(55,5)$ & 48 & $(88,9)$ & 26 & $(86,8)$ & 110 & $(73,9)$ \\
\hline Sífilis & 27 & $(41,5)$ & 3 & $(5,6)$ & 1 & $(3,3)$ & 31 & $(20,9)$ \\
\hline Rubéola & & - & 1 & $(1,8)$ & 1 & $(3,3)$ & 2 & $(1,3)$ \\
\hline Dengue & 1 & $(1,5)$ & & - & 1 & $(3,3)$ & 2 & $(1,3)$ \\
\hline Varicela & 1 & $(1,5)$ & & - & 1 & $(3,3)$ & 2 & $(1,3)$ \\
\hline Sida** & & - & 2 & $(3,7)$ & & - & 2 & $(1,3)$ \\
\hline Total & 65 & $(100,0)$ & 54 & $(100,0)$ & 30 & $(100,0)$ & 149 & $(100,0)$ \\
\hline
\end{tabular}




\begin{tabular}{|c|c|c|c|c|c|c|c|c|}
\hline \multirow{3}{*}{ Achados fundoscópicos } & \multicolumn{6}{|c|}{ Maternidade } & & \\
\hline & \multicolumn{2}{|c|}{ CISAM } & \multicolumn{2}{|c|}{ HBL } & \multicolumn{2}{|c|}{ HAM } & \multicolumn{2}{|c|}{ Total } \\
\hline & $\overline{\mathbf{N}}$ & $\%$ & $\overline{\mathbf{N}}$ & $\overline{\%}$ & $\mathbf{N}$ & $\%$ & $\mathbf{N}$ & $\%$ \\
\hline \multicolumn{9}{|l|}{ Alterações da retina } \\
\hline Hemorragias & 94 & 30,6 & 98 & 41,8 & 63 & 49,6 & 255 & 38,2 \\
\hline Atrofia EPR* & 15 & 4,9 & 7 & 3,0 & 1 & 0,8 & 23 & 3,4 \\
\hline Atrofia coriorretiniana & 1 & 0,3 & 2 & 0,8 & - & - & 3 & 0,4 \\
\hline Alterações do nervo óptico** & 6 & 1,9 & 6 & 2,4 & 1 & 0,8 & 13 & 1,9 \\
\hline Alterações vasculares*** & 4 & 1,3 & - & - & 1 & 0,8 & 5 & 0,7 \\
\hline Exame Normal & 187 & 61,0 & 122 & 52,0 & 61 & 48,0 & 370 & 55,4 \\
\hline Total & 307 & 100,0 & 235 & 100,0 & 127 & 100,0 & 669 • & 100,0 \\
\hline
\end{tabular}

multissistêmicas $^{(1,8,10)}$. No presente estudo, leucocoria com catarata congênita foi identificada em cinco olhos, no entanto, casos novos podem surgir com seguimento dos recém-nascidos encaminhados, principalmente os suspeitos de infecção congênita. Para identificação da leucocoria é realizado o exame do reflexo vermelho pupilar, muitas vezes dificultado pelo choro do neonato ${ }^{(8)}$, podendo algumas vezes confundir o examinador. Neste estudo, apesar de ter sido descrita ausência de reflexo vermelho em $3,4 \%$, o diagnóstico de leucocoria ocorreu em apenas $0,4 \%$ dos olhos.

A conjuntivite neonatal é uma condição potencialmente séria, tanto pelos efeitos locais ao olho, quanto pelo risco de disseminação sistêmica. Os fatores predisponentes às conjuntivites neonatais são parto vaginal, asfixia neonatorum e ruptura prolongada da membrana amniótica ${ }^{(13)}$. Todos os recémnascidos que apresentaram aspecto clínico de conjuntivite foram encaminhados para seguimento e investigação etiológica na FAV. Apesar da importância de investigar o agente microbiológico ${ }^{(13)}$, observou-se o uso rotineiro de colírio de cloranfenicol, prescrito pelos pediatras nos casos suspeitos de conjuntivite, sem qualquer investigação etiológica. Diante disso, os autores optaram por doar o colírio de tobramicina, com o intuito de abranger os patógenos mais freqüentes, visto que se trata de uma população de baixa renda, com dificuldades financeiras de iniciar o tratamento, e na tentativa de cobrir os casos que não dariam seguimento ambulatorial, talvez por dificuldades de transporte ou mesmo ignorância dos responsáveis.

A incidência de hemorragia retiniana pode variar de 2,6\% a $59,0 \%$, de acordo com a época em que é realizado o exame, ocorrendo a resolução da maioria destas já nos primeiros três dias de $v i^{(18)}$. Na presente casuística, foram identificadas hemorragias retinianas em 7,8\% do total de recém-nascidos examinados durante a primeira semana de vida, observando-se maior frequiência na maternidade do HBL. Também foi identificado nesta maternidade maior número de recém-nascidos prematuros e maior frequiência de uso da oxigenoterapia, podendo por isso estar relacionado às hemorragias, algum grau de hipóxia neonatal.
A grande variedade de examinadores, a inexperiência no exame oftalmológico do recém-nascido, além das próprias limitações do exame, podem ter contribuído para falhas na detecção ou hipervalorização de alguns achados no presente estudo. Algumas informações não foram identificadas, como o tipo de parto e Apgar, devido à dificuldade de acesso a alguns prontuários médicos, o mau preenchimento destes, e a ausência de algumas gestantes no leito do recém-nascido, que poderiam dar informações úteis, o que refletiu em falhas na análise dos dados coletados. Apesar de ter sido realizada uma triagem nos recém-nascidos, o encaminhamento dos casos suspeitos às diversas subespecialidades na FAV proporcionou melhores subsídios para a definição dos diagnósticos e suporte aos casos.

A determinação da incidência e prevalência das diversas causas de baixa visual na infância, é importante tanto para o desenvolvimento de estratégias de prevenção, como para o planejamento de futuras intervenções precoces ${ }^{(6)}$. Embora se admita a gravidade da perda da capacidade visual, especialmente em termos das conseqüências sociais e econômicas que acarreta, ainda há muito por se fazer mundialmente ${ }^{(14)}$.

Finalizando, salienta-se que a cegueira, além de afetar a pessoa física, psíquica, econômica e culturalmente, muda a dinâmica da família e afeta toda a sociedade, devendo-se portanto, tudo fazer para preveni-la ${ }^{(1)}$.

\section{AGRADECIMENTOS}

À Professora Sylvia Lemos Hinrichsen e ao Professor Natal pela orientação metodológica e estatística.

\section{ABSTRACT}

Purpose: To identify the main ocular diseases in a neonate population attended by the "Sistema Único de Saúde" in reference maternities of Pernambuco State, advising the follow-up and necessary treatment. Methods: A transversal study was done among newborn infants in three public maternities, 
during the period of April to October 2000. An ophthalmological examination was performed during weekly visits, advising the follow-up and treatment of the cases with ocular alterations or risk factors. Results: 3280 newborns were examined: 1403 (42.8\%) in the Encruzilhada maternity (CISAM), $1232(37.5 \%)$ in the maternity of the Hospital Barão de Lucena (MHBL) and $645(19.7 \%)$ in the maternity of the Hospital Agamenon Magalhães (MHAM). 387 were pre-term (11.8\%). 701 neonates $(21.4 \%)$ with ocular alterations or risk factors were referred to the specialized service. Of these, $46.4 \%$ were pre-term and $37.9 \%$ received oxygen therapy, with a higher percentage in MHBL (45.4\%). Conjunctivitis was found in $3.0 \%$ of the eyes, leucocoria in $0.4 \%$ and subconjunctival hemorrhage in $2.0 \%$. At fundoscopy, retinal hemorrhages were found in $7.8 \%$ of cases, with $4.3 \%$ affecting the macula. Conclusions: The most frequent diseases were retinal hemorrhages and conjunctivitis. The main risk factors seen were prematurity and neonatal infectious diseases. The authors suggest that ocular examinations should be done routinely in neonates.

Keywords: Eye diseases/pathology; Eye/pathology; Infant, newborn; Risk factors

\section{REFERÊNCIAS}

1. Adam Netto A, Peres SO. Catarata na infância- estudo de 106 casos. Rev Bras Oftalmol 1998;57:903-8.

2. Foster A, Gilbert C. Epidemiology of childhood blindness. Eye 1992;6:173-6.
3. Brito PR, Veitzman S. Causas de cegueira e baixa visão e crianças. Arq Bras Oftalmol 2000;63:49-54.

4. Lucena AR, Cantanhede TMI, Trigueiro SA, Tavares S, Ventura LO. Freqüência e causas da ambliopia em pacientes assistidos na Fundação Altino Ventura- Recife-PE. Rev Bras Oftalmol 2001;60:50-4.

5. Temporini ER. Pesquisa de oftalmologia em Saúde Pública: considerações metodológica sobre fatores humanos. Arq Bras Oftalmol 1991;54:279-81.

6. DeCarlo DK, Nowakowski R. Causes of visual impairment among students at the Alabama School for the blind. J Am Optom Assoc 1999;70:647-52.

7. Rahi JS, Dezateux C. National cross sectional study of detection of congenital and infantile Cataract in the United Kingdom: role of childhood screening and surveillance. BMJ 1999;318:362-5.

8. Tor Flage KB, Arnijot HM. Lukokori (hvit pupill) hos barn mor har alltid rett. Tidsskr Nor Loegeforen 1999;119:794-5.

9. Ventura M. Catarata congênita. In: Resende F. Cirurgia de catarata. Rio de Janeiro: Cultura Médica; 2000. p.399-408.

10. Cassidy L, Taylor D. Congenital cataract and multisystem disorders. Eye 1999; 13:464-73.

11. Sarquis ALF, Moreira Jr. CA, Ceccatto MP, Bagatin AC, Arrata KM. Retinopatia da prematuridade: análise dos casos num período de quatro anos. Pediatria (São Paulo) 1999;21:202-7.

12. Isenberg SJ, Apt L, Wood M. A controlled trial of povidone-iodine as prophylaxis against ophthalmia neonatorum. N Engl J Med 1995;332:562-6.

13. Iroha EO, Kesah CN, Egri-Okwaji MTC, Odugbemi TO. Bacterial eye infection in neonates, a prospective study in a neonatal unit. West Afr $\mathrm{J}$ Med 1998;17:168-72.

14. Temporini ER, Kara-José N. Níveis de prevenção de problemas oftalmológicos: propostas de investigação. Arq Bras Oftalmol 1995;58:189-92.

15. Leal DB, Tavares SS, Ventura LO, Florêncio T. Atendimento a portadores de visão subnormal: estudo retrospectivo de 317 casos. Arq Bras Oftalmol 1995;58:439-42.

16. Furtado F. Participação pediátrica em duas grandes causas de cegueira e medidas preventivas. Rev Bras Oftalmol 1988;47:302-6.

17. Ventura LO, Leal DB, Tavares SS, Araújo A, Linhares O, Gonçalves S. Catarata congênita bilateral: estudo comparativo dos achados clínicos observados em dois grupos pertencentes a níveis sócio-econômicos distintos em Pernambuco. Arq Bras Oftalmol 1995;58:429-3.

18. Kaur B, Taylor D. Fundus hemorrhages in infancy. Surv Ophthalmol 1992; 37:1-17.

Nos artigios enviados para publicação, o nome dos autores e suas afiliações devem estar completos. Isso facilitará a indexação e os links com as bases de dados e o CV Lates. 